\title{
Blockchain Technology in Supply Chain Management: An Application Perspective
}

\author{
Gregor Blossey \\ German Graduate School of \\ Management and Law, \\ Heilbronn, Germany \\ gregor.blossey@ggs.de
}

\author{
Jannick Eisenhardt \\ German Graduate School of \\ Management and Law, \\ Heilbronn, Germany \\ jannick.eisenhardt@studmail.ggs.de
}

\author{
Gerd J. Hahn \\ German Graduate School of \\ Management and Law, \\ Heilbronn, Germany \\ gerd.hahn@ggs.de
}

\begin{abstract}
Given the hype around the cryptocurrency Bitcoin, blockchain technology (BCT) has also received considerable attention outside the financial sector. Multiple applications of BCT in supply chain management (SCM) are discussed in business practice and there is increasing interest in this topic within the academic community. In this paper, we intend to combine these two perspectives on BCT in SCM to summarize a current state of the art and to derive avenues for further research. For this purpose, a comprehensive framework of use case clusters of $B C T$ in SCM is developed according to the distinctive features of BCT. The framework is used to analyze 53 applications of BCT in SCM which are derived from a systematic literature review and a secondary dataset of blockchain-driven innovations in SCM. We identify five emerging use case clusters of BCT in SCM which clearly extend the scope beyond frequently mentioned applications such as product tracking and tracing.
\end{abstract}

\section{Introduction and Research Background}

Introduced by one or more individuals under the pseudonym Satoshi Nakamoto [1], the cryptocurrency Bitcoin and the underlying blockchain technology (BCT) have created a tremendous hype around electronic payment systems using the peer-to-peer paradigm of the internet $[2,3]$. More generally, BCT provides the infrastructure that enables secure direct exchange of value between participants without any financial intermediary ('internet of value') [4].

Blockchain is a shared ledger that allows for immutable storage of verified transaction data [5]. Therefore, The Economist called blockchain the 'trust machine' [6] since a ledger most fundamentally represents a trustworthy record of business activity. Trust in business relationships is a key ingredient for inter-company supply chain (SC) collaboration [7, 8]. Consequently, supply chain management (SCM) is generally viewed as a major field of application for BCT which is confirmed by a series of recent news items [9]. Most notably, IBM and Maersk announced a joint venture to commercialize BCT in container shipping and global trade activities [10].

In the academic community, the interest in $\mathrm{BCT}$ and its business applications has been steadily growing over the last years [11] which is confirmed by a series of recent literature reviews (see [12, 13, 14, 15, 16]). An overarching research framework for BCT is provided by Risius and Spohrer [17]. In the slipstream of this development, SCM has only attracted minor interest among various application domains (see [18]).

While Korpela et al. [19] focus on the issue of data integration in digital SCs using BCT, Sternberg and Baruffaldi [20] provide a first summary of potential applications of BCT in SCM from the literature. Hackius and Petersen [21] and Petersen et al. [9] use an expert survey of logistics professionals to explore potential applications and future prospects of BCT in SCM. Petersen et al. [9] synthesize application clusters of BCT in logistics and SCM from publicly available case examples. To the best of our knowledge, there is no comprehensive perspective on use cases of BCT in SCM. We intend to fill this gap with this paper.

The contribution of this paper is twofold: first, a comprehensive framework is developed positioning BCT and its potential areas of application in the domain of SCM. In contrast to previous studies, this paper uses a deductive approach mapping distinctive features of BCT to use cases in SCM. Second, the framework is applied to the results of a structured literature review 
and a secondary dataset of BCT-enabled SC innovations. This allows for determining a current state of the art of BCT-enabled applications in SCM and for identifying future perspectives in this field.

The remainder of this paper is structured as follows: in section 2, we provide conceptual foundations of SCM and $\mathrm{BCT}$ and derive the corresponding framework of use case clusters. The systematic literature review and the analysis of the secondary dataset are conducted in sections 3 and 4 . The findings are discussed in section 5 . Section 6 concludes the paper with a summary of the findings and an outlook for further research.

\section{Conceptual Foundations}

\subsection{Supply Chain Management}

SCM covers two major tasks according to the Council of Supply Chain Management Professionals (CSCMP) [22]: (i) the planning, implementing, and controlling of primary activities that create and deliver value for the ultimate customer (esp. procurement, manufacturing, and logistics), and (ii) the integration and coordination of corresponding business processes within and across companies. While integration refers to the managerial and organizational challenges of forming a network of mostly independent companies, coordination is concerned with 'technical' implementation of processes and systems to foster alignment of material, financial, and information flows along the SC [8].

The importance of information and communication technology for SCM is widely acknowledged [23, 8]. Technology represents a major driver and constitutive element of SC innovation [24]. Recent advances as part of the Fourth Industrial Revolution (aka 'Industry 4.0 ' or 'Industrial Internet of Things') promise radical changes for various sectors including manufacturing and logistics [25]. Industry 4.0 envisions intelligent and connected physical assets, i.e., smart products and machines that operate autonomously and that can form self-coordinating systems such as smart factories or smart SCs [26]. In the slipstream of these developments, $\mathrm{BCT}$ and its implications on SCM receive increasing attention $[21,20]$.

\subsection{Blockchain Technology}

Most fundamentally, a blockchain is a distributed ledger that is shared and agreed upon a peer-to-peer network [27, 20]. A blockchain contains a single record of the data which is stored in blocks on every participant's node [12]. Each block corresponds to a timestamped record that is verified through a defined consensus protocol of the blockchain network and secured via public-key cryptography ('hashing') [13]. This basically eliminates the need for a trusted central entity. Since blocks are chained via their hash codes, information on the blockchain is immutable [28] and thus allows the user to obtain provenance information and to trace status changes over time [29].

A blockchain may execute computational logic in the form of 'smart contracts' (often referred to as chaincode) [2]. A smart contract is a trusted application that is installed on the nodes of the blockchain [30, 31]. With respect to access rights, permissionless and permissioned blockchains can be distinguished [32]. Both of these types can be either private or public depending on the ownership over data and infrastructure [33]. There are two dominant types of blockchains: permissionless-public blockchains are freely accessible via the internet (e.g. Bitcoin blockchain). In permissioned-private blockchains (e.g. Hyperledger Fabric) [28, 31], however, users need to register and are granted access by a network administrator based on a pre-defined approval process [5].

Although BCT enforces transparency, it allows for pseudonymity since transactions are settled between 30-plus-character alphanumeric addresses [2]. BCT is not restricted to financial transactions related to Bitcoins; any asset - both tangible (physical good) and intangible (property right or financial claim) - that entails value can be transfered onto or off the ledger $[34,20]$. The digital representative of this asset on the blockchain is called a 'token' [35].

While blockchain is largely viewed as a general purpose technology that further increases productivity, various authors argue that BCT will change the industrial organization [4, 35]. In a similar vein, Davidson et al. [36] characterize blockchain as an institutional technology that fosters economic coordination. As a consequence, blockchains represent a novel type of institution that is different from markets, hierarchies (firms), and relational contracts as described by Williamson [37]. More precisely, blockchains constitute decentralized collaborative (or autonomous) organizations for value creation and exchange [38].

SCs are networks of independent companies that are constituted by relational contracts [39]. Relational contracts represent informal agreements that are "sustained by the value of the future relationship" [40] and thus trust becomes an essential factor of SC relationships [41]. However, a blockchain removes trust from the equation and is therefore fundamentally different from an SC taking an institutional viewpoint. For the purposes of this paper, we mainly focus on the impact of BCT as a general purpose technology in SCM. 


\subsection{Linking SCM and BCT}

BCT provides four key features that can enhance integration and coordination among the members of an SC [35]: (1) transparency, (2) validation, (3) automation, and (4) tokenization. Transparency relates to the shared ledger of information which is aggregated from various sources and participants of the blockchain. Immutability of records and consensus-based verification enable validation of information [42]. Automation refers to the opportunity to execute smart contracts based on verified information on the blockchain. BCT allows creation of tokens that represent a specific claim on any valuable asset and their exchange between blockchain members (tokenization) [43]. Enabled by these four key features of BCT, one can derive corresponding use case clusters in SCM that build on one another:

1. SC Visibility: One of the main causes for SC inefficiencies is poor end-to-end transparency which also leads to the so-called bullwhip effect [8]. BCT allows sharing real-time information about the location and status of an object between multiple SC members [9]. Given the opportunities of sensor technology and the Internet of Things $[26,44]$, any measurable condition such as product temperature in a cold chain or availability of technical equipment operating in the SC can be tracked. This improves data accuracy enhancing collaborative planning and execution as well as the implementation of preventive and reactive risk management measures [35].

2. SC Integrity: Given a shared ledger of transparent and immutable records, BCT provides the opportunity to trace assets back to their origin [9]. Provenance information to certify authenticity ensures integrity of assets involving both products and technical equipment. This could enforce responsible sourcing and allow detecting or even preventing product counterfeit and other fraudulent actions [35]. Applications could involve tracing of asset ownership after sale for warranty purposes. Furthermore, BCT eases paperwork in global trade by ensuring validity of freight documents, e.g. in customs clearance [21].

3. SC Orchestration: Combining transparency and validation with automation via smart contracts, one could envision SCs that operate highly automated based on pre-specified rules [45]. This increases speed and eases coordination since information and corresponding decisions or measures are propagated throughout the SC. More specifically in case of a machine failure, the machine could order the spare part from the supplier, request maintenance service, and inform downstream parties about expected delays. Another benefit of automation is ex-post enforceability of contracts, i.e., contractual parties cannot reverse their commitments [35]. Consequently, BCT provides the necessary foundation to extend the smart factory paradigm of Industry 4.0 to inter-company SCs.

4. SC Virtualization: Virtualization is a well-known approach in IT infrastructure management to increase utilization and flexibility of IT assets by creating a logical representation of physical hardware in software [46]. Tokenization of physical SC assets such as technical equipment and inventories follows a similar idea since there is another opportunity besides shifting acquisition/sale of SC assets to the blockchain. Claims on capacities or ordering options could be issued as tokens and circulated outside normal (bilateral) contractual relationships. Similar to the virtualization of IT hardware, this would allow for improved capacity utilization of SC assets since excess capacities could be monetized. Moreover, virtualization would increase contract flexibility and enable reallocation of corresponding risks in SCs in general.[35]

While the aforementioned use cases mainly focus on managing physical assets and material flows, cross-cutting applications that support the management of corresponding financial claims and financial flows are grouped in a separate use case cluster [35]:

5. SC Finance: Applications supporting financial SCM are a natural 'fit' for BCT given the close ties to cryptocurrencies and the important role of financial intermediaries in global trade $[9,47]$. Consequently, there are two types of applications: first, BCT eases the settlement of multi-party and multi-tier financial transactions in SCs that result from collaborative value creation of blockchain members [38, 35]. Second, transparent and validated records as well as automated transactions and tokenized financial claims simplify financing of working capital (including inventories and accounts receivable net of accounts payable [48]) from blockchain members which also lowers financing costs [47]. For this purpose, SC assets could be collaterialized by issuing corresponding financial claims using tokens [35]. 


\section{Systematic Literature Review}

\subsection{Approach}

We strictly followed the principles of Webster and Watson [49] to ensure methodological rigor of the systematic literature review (SLR). In accordance with their recommended approach, we followed a three-step process: first, we identified relevant keywords and conducted a database search. Second, we critically evaluated the articles obtained from the database search to select relevant papers, and we conducted a manual search to identify further related items. Third, the final set of papers was organized and summarized.

\subsection{Database Search and Paper Selection}

Based on the subject classification of the Journal Quality List (JQL) [50], we defined a set of renowned journals from four subject areas General Management \& Strategy, Innovation, Management Information Systems \& Knowledge Management, and Operations Research, Management Science, and Production and Operations Management. The journals were selected based on the rankings of the 'Association of Business Schools Academic Journal Quality' (ABS) and 'Verband der Hochschullehrer für Betriebswirtschaft JOURQUAL 3' (VHB). Considering that the field of blockchain-related research is still in its infancy, we decided for a wide range of journal quality. We included journals ranked as 'recognized' or better which corresponds to a minimum ranking of 1 (ABS) or $\mathrm{C}(\mathrm{VHB})$. This resulted in a set of 214 journals.

Furthermore, six conference proceedings from the IS community were included: Proceedings of the Hawaii International Conference on System Sciences (HICSS), Proceedings of the European Conference on Information Systems (ECIS), Proceedings of the International Conference on Information Systems (ICIS), Proceedings of the Pacific Asia Conference on Information Systems (PACIS), Proceedings of the Internationale Tagung Wirtschaftsinformatik (WI), and Proceedings of the American Conference on Information Systems (AMCIS).

For the search, we require the term 'blockchain' and an SC-related keyword to appear in either title or abstract (including truncations of the respective search terms). The list of SC-related keywords contains 13 items and was determined based on the SCM definition of the CSCMP [22]: 'supply chain', 'network', 'business process', 'operations', 'procurement', 'sourcing', 'manufacturing', 'production', 'logistics', 'transportation', 'distribution', and 'warehouse'.
The resulting search string was applied to a meta-search engine (based on 202 different databases such as EBSCO Business Source Complete) given the aforementioned set of journals and to the AIS electronic Library (AISeL) to cover the conference proceedings. We did not impose a time restriction and limited the search to papers written in English. We obtained 61 results as of June 15th, 2018. This relatively low number of papers can be attributed to the early stage of the research in this field.

We constrained the SLR to full papers and excluded articles that did not discuss applications of BCT in SCM. This left us with a total of seven relevant articles. Based on this set of papers, a manual backward and forward search was conducted in order to identify further relevant articles which were not covered in the database search. This process yielded another ten articles resulting in 17 papers in total. The complete collection of papers is presented in Table 1.

\begin{tabular}{llc} 
Search type & References & Count \\
\hline Database & {$[51][19][52][53][54]$} & \\
& {$[55][20]$} & 7 \\
Manual & {$[56][42][57][58][21]$} & \\
& {$[59][60][61][62][63]$} & 10 \\
\hline
\end{tabular}

Table 1. Results of the SLR

\subsection{Paper Categorization}

Our search processes included 220 different outlets (214 journals and six conference proceedings). While 13 outlets contained relevant literature, only the Proceedings of the Hawaii International Conference on System Sciences contained more than one article (in total three). The earliest paper we found on BCT in SCM was published in 2016. Given four published articles in 2016, seven articles in 2017, and six articles in 2018 (as of June 15, 2018), it is apparent that this research stream is still in its infancy.

The sample includes six papers published in journals (35\%) while the remaining eleven were published in conference proceedings (65\%). The vast majority of the articles used conceptual approaches $(65 \%)$ describing potential applications of BCT and their implementation. Three studies applied qualitative empirical methods such as expert interviews while the remaining three studies used modeling-based approaches involving the Ethereum blockchain. Articles that use quantitative empirical research methods did not appear in the sample.

The set of papers in focus of this study informs about 29 applications. Food products (e.g. [59]) and container shipping (e.g. [21]) account for more than half of the 
cases and typically involve track and trace applications. Use cases in discrete manufacturing (e.g. [52]), the pharmaceutical industry (e.g. $[55,21]$ ), and mining (e.g. $[55,63])$ are only of minor importance in the sample. The results are summarized in Table 2.

\begin{tabular}{lr} 
Industry & Count \\
\hline Food products & $9(31 \%)$ \\
Container shipping & $7(24 \%)$ \\
Discrete manufacturing & $4(14 \%)$ \\
Pharmaceuticals & $3(10 \%)$ \\
Mining industry & $2(7 \%)$ \\
Other/not specified & $4(14 \%)$ \\
\hline & $\mathbf{2 9 ( 1 0 0 \% )}$
\end{tabular}

Table 2. Split of applications across industries

In a next step, we mapped the applications to the five use case clusters as introduced above. For this purpose, we focused on the dominant feature acknowledging that applications may fall into multiple categories. This is especially true for the clusters SC visibility and integrity that are closely interlinked and typically represent the foundation for the more advanced uses cases. To ensure inter-rater reliability, the classification was performed by two authors independently; conflicts were resolved involving the third author. Table 3 summarizes the results for the respective use case clusters.

\begin{tabular}{lr} 
Use case cluster & Count \\
\hline Visibility & $3(10 \%)$ \\
Integrity & $13(45 \%)$ \\
Orchestration & $13(45 \%)$ \\
thereof including SC finance aspects & $6(21 \%)$ \\
Virtualization & $0(0 \%)$ \\
\hline & $\mathbf{2 9}(\mathbf{1 0 0 \%})$
\end{tabular}

Table 3. Summary of use case clusters (SLR)

Applications discussed in the literature largely focus on the issue of SC integrity (45\%) and SC orchestration $(45 \%)$ while specific applications that solely cover SC visibility are relatively scarce (10\%). There are no applications in the sample that capture the benefits of SC virtualization. Applications that foster SC visibility facilitate information exchange between SC parties [62] and enable accurate tracking of materials and products along the SC [20, 60] which is the prerequisite for implementing counter-measures if required conditions are violated.

There are two dominant groups of applications in the cluster $S C$ integrity. The first group relates to applications that support traceability for food safety. Having a complete and transparent chain of custody allows to detect or even prevent fraudulent activities and enables authorities to take faster action in response to food-borne diseases (e.g. [57, 55, 20]). BCT can also be used to verify the provenance of luxury goods such as diamonds [55]. Similar requirements emerge from applications that support ethical sourcing or tracing of conflict minerals. The second largest group covers applications that originate from international shipping and that aim at digitizing extensive paperwork (esp. related to the bill of lading) in order to reduce errors and fraud $[53,55,42,21]$.

Applications for SC orchestration are oftentimes closely connected to tracking and tracing applications (e.g. [58]) such that further processes are automatically triggered once a status is confirmed or sensors detect a certain condition [56, 42, 53]. For instance, Kshetri et al. [55] present an application that supports pharmaceutical companies in monitoring transportation conditions of temperature-sensitive products. If the required conditions are not fulfilled, release of the pharmaceutical products is prohibited immediately. Nearly half of the SC orchestration applications include a SC finance component. In these cases, smart contracts trigger financial payments (e.g. $[19,21]$ ) or facilitate financing of working capital between SC partners [9].

\section{Current Trends of BCT-enabled Applications in SCM}

To investigate current trends of BCT in SCM, we use a dataset of BCT-enabled SC innovations which are derived from the startup and venture capital platform angel.co [64]. A list of 515 startup companies was extracted from the categories 'blockchain' and 'blockchain / cryptocurrency' as of June 15th, 2018. We only included companies with at least a moderate performance rating on the platform (corresponding to a 'signal strength' of 3 out 5 or higher).

Startup companies that did not have an SC-related tag or where the description did not contain SC content were removed. This especially relates to financial payment systems in global trade and asset-based lending platforms that were not attached to SC processes. We ended up with 24 companies of which 54\% (13) are located in the Americas, 33\% (8) in Europe, and $13 \%$ (3) in Asia. The companies have joined the platform since January 2012 and are listed on average for 34 months. Four out of the corresponding 24 applications have a clear industry focus: fashion, retail, and pharmaceuticals $(2 \mathrm{x})$.

The 24 applications were classified according to the five BCT-enabled uses case clusters in SCM as introduced above. In case SC finance matters are attached to applications, we have flagged the respective 
cases accordingly. To ensure inter-rater reliability, the classification was performed by two authors independently; conflicts were resolved involving the third author. The results are summarized in Table 4.

\begin{tabular}{lr} 
Use case cluster & Count \\
\hline Visibility & $2(8 \%)$ \\
Integrity & $7(29 \%)$ \\
Orchestration & $8(34 \%)$ \\
thereof including SC finance aspects & $2(8 \%)$ \\
Virtualization & $7(29 \%)$ \\
thereof including SC finance aspects & $3(12 \%)$ \\
\hline & $\mathbf{2 4 ( 1 0 0 \% )}$
\end{tabular}

Table 4. Summary of use case clusters (startups)

Applications that solely focus on SC visibility only account for $8 \%$ while SC integrity and SC orchestration take a major share of $29 \%$ and $34 \%$ of the applications, respectively. This observation shows that startup companies extend their offerings beyond supporting basic transparency in the SC which in turn is a prerequisite for the more advanced use cases. The solutions for SC visibility support information sharing between SC members in fashion and retail industries. Solutions that ensure $S C$ integrity can be divided into two groups. While four solutions focus on anti-counterfeiting and provenance tracing, three solutions support electronic document management with respect to requirements of global trade (related to the bill of lading) or industry-specific regulatory requirements such as within the pharmaceutical industry. The main motivation behind these projects are cost reductions through efficiency gains. One reason is that many inter-company SC processes are primarily paper-based which leads to high costs in aggregation and verification of information.

Automated SC orchestration represents an important use case cluster which can be attributed to the emerging paradigm of Industry 4.0 and novel applications of sensor technology due to the Internet of Things. Smart contracts play a pivotal role for automating SC operations by enabling machine-to-machine interaction. We found two specific applications that integrate $S C$ finance into SC orchestration. One case is concerned with machine-to-machine interaction involving both the orchestration of material and financial flows. The second solution implements automated billing and payments across the SC. Consequently, BCT provides the missing building block to enable smart SC operations extending beyond firm boundaries.

The tokenization of assets enables $S C$ virtualization. Corresponding applications in the sample provide opportunities for smart contract-enabled distributed manufacturing, i.e., tokenized ('virtual') manufacturing capacities can be traded between blockchain members. The same approach can also be applied to better utilize excess capacities in logistics. Similar ideas have already been discussed in the SC context with the concept of the so-called Physical Internet [65] that involves shared and highly automated SC infrastructure. Three applications for SC virtualization in the sample also integrate aspects of $S C$ finance enabling asset-based lending and trade financing between SC partners.

\section{Discussion}

In this section, we summarize a current state of the art of BCT in SCM and develop perspectives for further research. Investigating the applications, we observe that tracking and tracing are natural use cases given the fundamental benefit of the shared ledger paradigm. Consequently, applications in document-intensive contexts such as global trade and industries that are subject to strict regulatory requirements such a pharmaceuticals and food products are prevailing. However, novel use cases emerge in combination with digital technologies [66] that enable automated orchestration of SC operations.

The next evolutionary development step will be on-demand SCs that make use of virtual ('tokenized') $\mathrm{SC}$ assets. This development largely corresponds to the ongoing service transformation of the industrial sector [25]. However, this still represents an intermediate step towards fully digitalized SCs as proposed in the literature (see [19]). Given apparent similarities to the concept of the Physical Internet which also applies the principles of distributed networks to the design and operation of SCs, a thorough delineation of both concepts and an investigation of potential synergies could be subject of further research.

Given the close ties of BCT and cryptocurrencies, we would have expected more applications in the SC finance cluster. While electronic payment systems seem to be well-established and account for the majority of BCT-enabled startups [64], financing activities such as asset-based lending are mostly decoupled from SC activities. However, SC finance and especially trade financing are a huge market involving more than 12 tn USD in trade credits in export business [35].

Applications of BCT mostly focus on efficiency improvements and risk mitigation from a single-firm perspective. This largely corresponds to the viewpoint of BCT being a general purpose technology that omits the benefits of improved economic coordination due to novel institutional arrangements. More specifically, SCs could be fundamentally reorganized 
as blockchain networks that enable collaborative value creation and thus allow capturing the value potential of comprehensive ecosystems. Consequently, further research should examine novel ecosystem-based business models and corresponding use cases that could be enabled by BCT.

BCT has the potential to enhance existing SC processes substantially and may lead to entirely new organizational structures in the future. However, one has to acknowledge several issues that need to be addressed in order to provide a comprehensive perspective on this technology in the SC context. Even though information on the blockchain is immutable, the initial data input is prone to errors resulting into the 'garbage in, garbage out' problem [35]. Moreover, there is no guarantee that the physical counterpart of the data has not been tampered with [67]. Consequently, BCT may improve business processes but will not make quality checks and audits obsolete given poor data quality in SCs. Another field that requires further investigation relates to the issue of data ownership in an open and distributed system such as a blockchain [20].

\section{Conclusion and Outlook}

In this paper, we investigated applications of BCT in and their benefit to SCM. For this purpose, a comprehensive framework of use case clusters was developed that correspond to key features of BCT. We used the framework to analyze 53 applications of BCT in SCM that were derived from both the literature and business practice. This enabled us to describe a current state of the art and to develop future perspectives.

The findings of this paper can be summarized as follows: first, applications of BCT in SCM can be grouped into five use case clusters. Dominant use cases cover provenance tracing of assets as well as automation of SC operations while aspects of SC finance are only supported occasionally in blockchain applications. Virtual on-demand SCs will form the next evolutionary step of BCT in SCM which coincides with the ongoing service transformation of the industrial sector. Second, applications of BCT solely focus on capturing the benefits of implementing a novel general purpose technology. However, this perspective largely omits the institutional innovation potential of blockchains reorganizing SCs for collaborative ecosystem-based value creation. Third, BCT is subject to several technical limitations which are especially relevant for SC-oriented use cases. These issues relate to the governance model of data ownership and typically low data quality in SC settings.

Given the early stage of this field of research, conclusions need to be seen as preliminary. The limitations of this study pertain mostly to the sample of applications and the methodological approach. This paper uses two different sources including 53 applications to provide extensive evidence and to reduce the potential for bias. The generalizability of the findings, however, depends on the scope of applications selected. Although a rigorous approach to content analysis was applied to ensure the reliability and validity of the results, interpreting the results depends on the coder's individual assessment of the data.

This paper mainly focused on the issues of manufacturing and logistics within SCM. However, there is a multitude of BCT-enabled applications in related fields of service operations management such as healthcare and retailing. Consequently, a similar study could be conducted for these industries to extend the scope to the broader context of operations management. Furthermore, there is only few literature applying empirical research methods; especially quantitative approaches are scarce. Corresponding approaches could be used to gain insight into realized benefits as well as success factors and challenges of implementing blockchain applications in the SC context.

\section{References}

[1] S. Nakamoto, "Bitcoin: A peer-to-peer electronic cash system." Available at https://bitcoin.org/bitcoin.pdf, retrieved 06/15/2018, 2008.

[2] M. Iansiti and K. R. Lakhani, "The truth about blockchain," Harvard Business Review, vol. 95, no. 1, pp. 118-127, 2017.

[3] C. Mulligan, J. Zhu Scott, S. Warren, and J. Rangaswami, "Blockchain beyond the hype: A practical framework for business leaders." World Economic Forum, Cologny/Geneva, 2018.

[4] D. Tapscott and A. Tapscott, "How blockchain will change organizations," MIT Sloan Management Review, vol. 58, no. 2, pp. 10-13, 2017.

[5] M. Pilkington, "Blockchain technology: Principles and applications," in Research Handbook on Digital Transformations (F.-J. Olleros and M. Zhegu, eds.), pp. 225-253, Cheltenham, UK: Edward Elgar Publishing, 2016.

[6] "The trust machine: The technology behind bitcoin could transform how the economy works," The Economist, $10 / 31 / 2015$.

[7] I.-W. G. Kwon and T. Suh, "Factors affecting the level of trust and commitment in supply chain relationships," Journal of Supply Chain Management, vol. 40, no. 1, pp. 4-14, 2004.

[8] H. Stadtler, "Supply chain management and advanced planning--basics, overview and challenges," European Journal of Operational Research, vol. 163, no. 3, pp. 575-588, 2005.

[9] M. Petersen, N. Hackius, and B. von See, "Mapping the sea of opportunities: Blockchain in supply chain and logistics.” Kühne Logistics University, Hamburg, 2017. 
[10] IBM, "IBM and Maersk to form joint venture to bring efficient, secure trade to global shipping industry." Available at https://www-03.ibm.com/press/us/en/photo/53603.wss, retrieved 06/15/2018, 2018.

[11] S. Miau and J.-M. Yang, "Bibliometrics-based evaluation of the blockchain research trend: 2008 - march 2017," Technology Analysis \& Strategic Management, vol. 30, no. 9, pp. 1029-1045, 2018.

[12] J. Yli-Huumo, D. Ko, S. Choi, S. Park, and K. Smolander, "Where is current research on blockchain technology? A systematic review," PloS one, vol. 11, no. 10, p. e0163477, 2016.

[13] S. Seebacher and R. Schüritz, "Blockchain technology as an enabler of service systems: A structured literature review," in Exploring Services Science (S. Za, M. Dragoicea, and M. Cavallari, eds.), pp. 12-23, Springer International Publishing, 2017.

[14] J. Lindman, V. K. Tuunainen, and M. Rossi, "Opportunities and risks of blockchain technologies: A research agenda," in Proceedings of the 50th Annual Hawaii International Conference on System Sciences 2017 (HICSS-50), pp. 1533-1542, 2017.

[15] Z. Zheng, S. Xie, H. Dai, X. Chen, and H. Wang, "Blockchain challenges and opportunities: A survey," International Journal of Web and Grid Services, 2018.

[16] Y. Li, T. Marier-Bienvenue, A. Perron-Brault, X. Wang, and G. Paré, "Blockchain technology in business organizations: A scoping review," in Proceedings of the 51 st Annual Hawaii International Conference on System Sciences 2018 (HICSS-51), pp. 4474-4483, 2018.

[17] M. Risius and K. Spohrer, "A blockchain research framework," Business \& Information Systems Engineering, vol. 59, no. 6, pp. 385-409, 2017.

[18] G. Salviotti, L. M. de Rossi, and N. Abbatemarco, "A structured framework to assess the business application landscape of blockchain technologies," in Proceedings of the 51st Annual Hawaii International Conference on System Sciences 2018 (HICSS-51), pp. 3467-3476, 2018.

[19] K. Korpela, J. Hallikas, and T. Dahlberg, "Digital supply chain transformation toward blockchain integration," in Proceedings of the 50th Annual Hawaii International Conference on System Sciences 2017 (HICSS-50), pp. 4182-4191, 2017.

[20] H. Sternberg and G. Baruffaldi, "Chains in chains: Logic and challenges of blockchains in supply chains," in Proceedings of the 51st Annual Hawaii International Conference on System Sciences 2018 (HICSS-51), pp. 3936-3943, 2018.

[21] N. Hackius and M. Petersen, "Blockchain in logistics and supply chain: Trick or treat?," in Digitalization in Supply Chain Management and Logistics (W. Kersten, T. Blecker, and C. M. Ringle, eds.), Proceedings of the Hamburg International Conference of Logistics (HICL), pp. 3-18, Berlin: epubli GmbH, 2017.

[22] CSCMP, "Supply Chain Management Terms and Glossary." Available at http://cscmp.org/CSCMP/Educate/SCM_Definitions_and _Glossary_of_Terms/CSCMP/Educate/SCM_Definitions_ and_Glossary_of_Terms.aspx, retrieved 06/15/2018, 2018.

[23] A. Gunasekaran and E. Ngai, "Information systems in supply chain integration and management," European Journal of Operational Research, vol. 159, no. 2, pp. 269-295, 2004.
[24] J. S. Arlbjørn, H. de Haas, and K. B. Munksgaard, "Exploring supply chain innovation," Logistics Research, vol. 3, no. 1, pp. 3-18, 2011.

[25] H. Kagermann, "Change through digitization: Value creation in the age of Industry 4.0," in Management of Permanent Change ( $\mathrm{H}$. Albach, H. Meffert, and A. Pinkwart, eds.), pp. 23-45, Wiesbaden: Springer Fachmedien, 2015.

[26] M. E. Porter and J. E. Heppelmann, "How smart, connected products are transforming competition," Harvard Business Review, vol. 92, no. 11, pp. 64-88, 2014.

[27] M. Crosby, Nachiappan, P. Pattanayak, S. Verma, and V. Kalyanaraman, "Blockchain technology: Beyond bitcoin," Applied Innovation Review, no. 2, pp. 6-19, 2016.

[28] K. Siba, Tarun, and A. Prakash, "Block-chain: An evolving technology," Global Journal of Enterprise Information System, vol. 8, no. 4, pp. 29-35, 2017.

[29] H. M. Kim and M. Laskowski, "Toward an ontology-driven blockchain design for supply chain provenance," Intelligent Systems in Accounting, Finance and Management, vol. 25, no. 1, pp. 18-27, 2018.

[30] N. Szabo, "Formalizing and securing relationships on public networks," First Monday, vol. 2, no. 9, 1997.

[31] E. Androulaki, Y. Manevich, S. Muralidharan, C. Murthy, B. Nguyen, M. Sethi, G. Singh, K. Smith, A. Sorniotti, C. Stathakopoulou, M. Vukolić, A. Barger, S. W. Cocco, J. Yellick, V. Bortnikov, C. Cachin, K. Christidis, A. de Caro, D. Enyeart, C. Ferris, and G. Laventman, "Hyperledger Fabric," in Proceedings of the Thirteenth EuroSys Conference, 2018.

[32] H. Wu, Z. Li, B. King, Z. Ben Miled, J. Wassick, and J. Tazelaar, "A distributed ledger for supply chain physical distribution visibility," Information, vol. 8, no. 4, p. 137, 2017.

[33] B. Carson, G. Romanelli, P. Walsh, and A. Zhumaev, "Blockchain beyond the hype: What is the strategic business value?." McKinsey \& Company, 2018.

[34] B. Notheisen, J. B. Cholewa, and A. P. Shanmugam, "Trading real-world assets on blockchain," Business \& Information Systems Engineering, vol. 59, no. 6, pp. 425-440, 2017.

[35] V. Babich and G. Hilary, "Distributed ledgers and operations: What operations management researchers should know about blockchain technology," SSRN Electronic Journal, 2018.

[36] S. Davidson, P. de Filippi, and J. Potts, "Blockchains and the economic institutions of capitalism," Journal of Institutional Economics, vol. 14, no. 4, pp. 639-658, 2018.

[37] O. E. Williamson, The Economic Institutions of Capitalism: Firms, Markets, Relational Contracting. New York, NY: Free Press, 1985.

[38] V. Schlatt, A. Schweizer, N. Nils Urbach, and G. Fridgen, "Blockchain: Grundlagen, Anwendungen und Potentiale." Fraunhofer-Institut für Angewandte Informationstechnik FIT, Bayreuth, 2016.

[39] A. Halldorsson, H. Kotzab, J. H. Mikkola, and T. Skjøtt-Larsen, "Complementary theories to supply chain management," Supply Chain Management: An International Journal, vol. 12, no. 4, pp. 284-296, 2007. 
[40] G. Baker, R. Gibbons, and K. J. Murphy, "Relational contracts and the theory of the firm," The Quarterly Journal of Economics, vol. 117, no. 1, pp. 39-84, 2002.

[41] B. S. Sahay, "Understanding trust in supply chain relationships," Industrial Management \& Data Systems, vol. 103, no. 8, pp. 553-563, 2003.

[42] K. Christidis and M. Devetsikiotis, "Blockchains and smart contracts for the internet of things," IEEE Access, vol. 4, pp. 2292-2303, 2016.

[43] Y. Chen, "Blockchain tokens and the potential democratization of entrepreneurship and innovation," Business Horizons, vol. 61, no. 4, pp. 567-575, 2018.

[44] R. Beck, M. Avital, M. Rossi, and J. B. Thatcher, "Blockchain technology in business and information systems research," Business \& Information Systems Engineering, vol. 59, no. 6, pp. 381-384, 2017.

[45] H. Diedrich, Ethereum: Blockchains, Digital Assets, Smart Contracts, Decentralized Autonomous Organizations. Lexington, KY: Wildfire Publishing, preview 3rd ed., 2016.

[46] K. C. Laudon and J. P. Laudon, Management Information Systems: Managing the Digital Firm. Harlow: Pearson Education Limited, fifteenth global ed., 2017.

[47] E. Hofmann, U. M. Strewe, and N. Bosia, Supply Chain Finance and Blockchain Technology: The Case of Reverse Securitisation. Cham: Springer International Publishing, 2018.

[48] E. Hofmann and H. Kotzab, "A supply chain-oriented approach of working capital management," Journal of Business Logistics, vol. 31, no. 2, pp. 305-330, 2010.

[49] J. Webster and R. T. Watson, "Analyzing the past to prepare for the future: Writing a literature review," $M I S$ Quarterly, vol. 26, no. 2, pp. xiii-xxiii, 2002.

[50] A.-W. Harzing, "Journal Quality List, 62nd edition, 3 April 2018,” 2018.

[51] I. Weber, X. Xu, R. Riveret, G. Governatori, A. Ponomarev, and J. Mendling, "Untrusted business process monitoring and execution using blockchain," in International Conference on Business Process Management, pp. 329-347, 2016.

[52] Y. Madhwal and P. B. Panfilov, "Industrial case: Blockchain on aircraft's parts supply chain management," in Americas Conference on System Science (AMCIS), 2017.

[53] K. Nærland, C. Müller-Bloch, R. Beck, and S. Palmund, "Blockchain to rule the waves: Nascent design principles for reducing risk and uncertainty in decentralized environments," in Proceedings of the 38th International Conference on Information Systems (ICIS), 2017.

[54] S. Hua, E. Zhou, B. Pi, J. Sun, Y. Nomura, and H. Kurihara, "Apply blockchain technology to electric vehicle battery refueling," Proceedings of Hawaii International Conference on System Sciences 2018 (HICSS-51), pp. 4494-4502, 2018.

[55] N. Kshetri, "1 blockchain's roles in meeting key supply chain management objectives," International Journal of Information Management, vol. 39, pp. 80-89, 2018.

[56] S. A. Abeyratne and R. P. Monfared, "Blockchain ready manufacturing supply chain using distributed ledger," International Journal of Research in Engineering and Technology, vol. 5, no. 9, pp. 1-10, 2016.
[57] F. Tian, "An agri-food supply chain traceability system for china based on RFID \& blockchain technology," in 13th International Conference on Service Systems and Service Management (ICSSSM), 2016.

[58] S. Chen, R. Shi, Z. Ren, J. Yan, Y. Shi, and J. Zhang, "A blockchain-based supply chain quality management framework," in 14th IEEE International Conference on E-Business Engineering, pp. 172-176, 2017.

[59] F. Tian, "A supply chain traceability system for food safety based on HACCP, blockchain \& internet of things," in 14th International Conference on Service Systems and Service Management (ICSSSM), 2017.

[60] Z. Gao, L. Xu, L. Chen, X. Zhao, Y. Lu, and W. Shi, "CoC: A unified distributed ledger based supply chain management system," Journal of Computer Science and Technology, vol. 33, no. 2, pp. 237-248, 2018.

[61] A. Imeri and D. Khadraoui, "The security and traceability of shared information in the process of transportation of dangerous goods," in Proceedings of the 9th IFIP International Conference on New Technologies, Mobility \& Security, 2018.

[62] Z. Li, W. M. Wang, G. Liu, L. Liu, J. He, and G. Q. Huang, "Toward open manufacturing: A cross-enterprises knowledge and services exchange framework based on blockchain and edge computing," Industrial Management \& Data Systems, vol. 118, no. 1, pp. 303-320, 2018.

[63] F. S. A. Pour, U. Tatar, and A. Gheorghe, "Agent-based model of sand supply governance employing blockchain technology," in Proceedings of the Annual Simulation Symposium 2018, 2018.

[64] Angel.co, "AngelList: Where the world meets startups." Available at https://angel.co, retrieved 06/15/2018, 2018.

[65] J. Mervis, "The information highway gets physical," Science, vol. 344, no. 6188, pp. 1104-1107, 2014.

[66] M. E. Porter and J. E. Heppelmann, "How smart, connected products are transforming companies," Harvard Business Review, vol. 93, no. 10, pp. 96-114, 2015.

[67] S. Apte and N. Petrovsky, "Will blockchain technology revolutionize excipient supply chain management?," Journal of Excipients and Food Chemicals, vol. 7, no. 3, pp. 76-78, 2016. 